\title{
Observation of Earth's Orbital Motion Using Cosmic-Ray Compton-Getting Effect at Matsushiro Underground Station
}

\author{
Shinichi Yasue ${ }^{1}$, Satoru Mori ${ }^{1}$, Kazuoki Munakata ${ }^{1}$, Alaa A. Darwish ${ }^{2}$, \\ and A. Adel Bishara ${ }^{2}$ \\ ${ }^{1}$ Department of Physics, Faculty of Science, Shinshu University, Matsumoto 390, Japan \\ ${ }^{2}$ Department of Physics, Faculty of Science, Alexandria University, Alexandria, Egypt
}

(Received May 16, 1991; Revised June 27, 1991)

\begin{abstract}
The Earth's motion Compton-Getting effect on the solar diurnal variation of cosmic ray intensity, has been examined by using observed data at Matsushiro underground station located at the effective vertical depth of 220 m.w.e. underground (threshold energy $\sim 7 \cdot 10^{10} \mathrm{eV}$, median primary energy $\gtrsim 7 \cdot 10^{11} \mathrm{eV}$ ) for the period 1980-1990. In the analysis, to eliminate the atmospheric effects on the muon intensity, the differences of the observed intensity between two-directional telescopes; the east-pointing (E-) and the west-pointing (W-) telescope, are used. Also the analysis is made for two different periods of the solar activity; the quiet period for averaged sunspot numbers $Z<60$ (1984-1988) and the active period for $Z$ $>60$ (1980-1984 and 1988-1989). It is shown that for the quiet period, the difference E-W diurnal vector has the phase of $22.5 \pm 1.3 \mathrm{hr}$ LT and the amplitude of $0.030 \pm 0.007 \%$. With a clockwise rotation of this $\mathrm{E}-\mathrm{W}$ vector by the right angle, we may compare it with that expected from the Earth's motion Compton-Getting effect. It is found that the observed $(0.030 \pm 0.007 \%$ in $4.5 \pm 1.3 \mathrm{hr} \mathrm{LT})$ and the expected vector $(\sim 0.021 \%$ in $\sim 6 \mathrm{hr} \mathrm{LT})$ are in fair agreement with each other. It is also found that for the active period, the observed E-W diurnal vector seems likely to show some contribution from the solar diurnal anisotropy, in addition to the Compton-Getting effect on it. This suggests that for the last solar active period the upper cut-off energy of solar diurnal anisotropy might be as high as that (several $\left.10^{11} \mathrm{eV}\right)$ for the threshold $\left(\sim 7 \cdot 10^{10} \mathrm{eV}\right)$ of 220 m.w.e. depth underground.
\end{abstract}

\section{Introduction}

The effect on the solar diurnal variation of cosmic ray intensity due to the Earth's orbital motion is well known as the Compton-Getting effect (hereafter abbreviated to the C-G effect), and it was first discussed by COMPTON and GETTING (1935) in 1935. The cosmic ray detector on the Earth's surface would observe an enhanced intensity when it looks along the direction of the Earth's movement with respect to the rest frame of the cosmic ray plasma. The fractional intensity enhancement should be for vertically incident cosmic rays, as

$$
\Delta I / I=(\gamma+2)(v / c) \cos \lambda
$$

where $\gamma$ is the exponent of a power law form $E^{-\gamma}$ of the cosmic ray differential energy spectrum, $v$ is the velocity of the Earth's orbital motion, $c$ the velocity of light, and $\lambda$ is the 
geographic latitude of the observation site. It is noted that the C-G effect should peak near 6 hr LT because of the Earth's orbital motion. So far, some studies of the cosmic ray effect to be observed from this effect have been reported. Among those studies, with an exception for an excellent evidence given by NAGASHIMA et al. (1989) by means of the east-west directional air-shower measurements (median primary energy, $E m \sim 10^{13} \mathrm{eV}$ ), the results at shallow underground ( $60 \sim 80$ m.w.e. depth, $E m=3 \sim 5 \cdot 10^{11} \mathrm{eV}$; DAVIES et al., 1979; FUJII and UENO, 1990) are influenced in a certain extent by the geomagnetic and solar modulated effects, and also the results by the uni-directional telescope at deep underground ( 429 m.w.e. depth, $E m \sim 2 \cdot 10^{12} \mathrm{eV}$; CUTLER and GROOM, 1986) are somewhat contaminated by the atmospheric effects. In this letter, we examine the C-G effect using observed cosmic ray muon data at Matsushiro underground station located at the effective vertical depth of 220 m.w.e. The present data have some advantages; first, the underground depth is deep, so the median primary energy of response $\left(E m \gtrsim 7 \cdot 10^{11} \mathrm{eV}\right)$ is high enough to be less influenced by the geomagnetic and solar effects. Secondly, the muon telescope used has multi-directional channels, which makes it possible to eliminate inherent atmospheric effects on the observed muons by taking the difference between the directional telescopes. Further, as the present period to be analyzed cover almost one solar cycle, we may also discuss the solar activity dependence of the observation.

\section{Analysis of Data}

Matsushiro underground station is located at Matsushiro-machi in Nagano Pref. Japan, at the geographic latitude $36.8^{\circ} \mathrm{N}$ and longitude $138.0^{\circ} \mathrm{E}$, whose effective vertical depth is 220 m.w.e. The muon telescope is constructed with the plastic scintillation detector $\left(1 \times 1 \mathrm{~m}^{2}\right.$ area each, viewed by double 5-in. photomultiplier tubes), arranged in two layers separated by $1 \mathrm{~m}$, with 16 detectors in the upper and 7 detectors in the lower due to its limited space in the tunnel. The telescope has multi-directional channels of the north-, the south-, the east-, and the west-direction with 9 channels totally, whose median primary energies of responses are ranged $E m=6 \cdot 10^{11} \sim 10^{12} \mathrm{eV}$. The continuous observation of the cosmic ray muon intensity has been made since August 1980 up to the present, whose duty cycle in operation is $\sim 95 \%$. The monitoring of the whole experimental system including data transmission has been made each hour via standard telephone lines from our remote cosmic-ray laboratory $(\sim 70 \mathrm{~km}$ in distance) in Matsumoto. Some characteristics of the telescope are shown in Table 1, where Etelescope is combined with two sub-telescopes of the northeast-pointing (NE-) and the southeast-pointing (SE-) telescope, and W-telescope with two sub-telescopes of the northwest-pointing (NW-) and the southwest-pointing (SW-) telescope, respectively. In more details of the station and the telescope, the reader may refer to our original paper (YASUE $e t$ al., 1979).

The hourly data (without any corrections) are Fourier-analyzed for both sidereal and solar frequencies. Some of the analyzed results for the sidereal frequency case are already reported elsewhere (YASUE et al., 1984, 1985). In the following analysis, solar activity sensitivity of the Compton-Getting effect is examined by dividing the present period covered almost one solar cycle (1980-1989) into two groups according to averaged sunspot numbers $Z$; the quiet (Q-) period for $Z<60$ (1984 Aug.-1988 Jul.), and the active (A-) period for $Z>60$ (1980 Aug.-1984 Jul. and 1988 Aug.-1989 Jul.). In the analysis, also the atmospheric effects or the malfunciton of the telescope which are common to the directional telescopes, are eliminated by taking the difference of the observed solar diurnal vectors between E- and W- 
telescope mentioned above (see Table 1). The difference method has been successfully applied to the air-shower data analysis by NAGASHIMA et al. (1989), but for the data analysis of the muon telescope the first application of the E-W method has been made in this work. Figure 1 shows yearly solar diurnal vectors and their difference E-W, plotted with filled circles for Qperiod and with open circles for A-period. In the figure, the averaged E-W vectors are also shown with the solid line for both Q- and A-period. The errors are derived from dispersion of yearly average. The numerical values of the observed $\mathrm{E}-\mathrm{W}$ vectors are given in Table 2 . We can clearly see in the figure and the table that the E-W vectors, particularly for Q-period, are statistically significant and that the vectors in the two periods are separated significantly from each other. Figure 2 shows these E-W vectors in a summation harmonic dial, illustrated with the solid line during Q-period (filled circles) and with the dotted line (open circles) during Aperiod. A persistency of these vectors may be evident, particularly for Q-period.

\section{Discussion and Conclusion}

The observed solar diurnal vector $\boldsymbol{D}(\mathrm{SO})$ can be regarded as a composition of three vectors; $\boldsymbol{D}(\mathrm{TEMP})$ due to the atmospheric effects, $\boldsymbol{D}(\mathrm{CR})$ due to cosmic ray diurnal anisotropy, and $\boldsymbol{D}(\mathrm{CG})$ due to the Earth's motion Compton-Getting effect (C-G effect), and

$$
D(\mathrm{SO})=\boldsymbol{D}(\mathrm{TEMP})+\boldsymbol{D}(\mathrm{CR})+\boldsymbol{D}(\mathrm{CG})
$$

Table 1. Muon telescope used in the analysis. E-telescope is combined with the northeast- (NE-) and the southeast-pointing (SE-) telescope, and W-telescope with the northwest- (NW-) and the southwestpointing (SW-) telescopes.

\begin{tabular}{|c|c|c|c|c|c|}
\hline Telescope & Comp. & $\begin{array}{c}\text { Viewing } \\
\lambda \mathrm{cd}\left({ }^{\circ}\right)\end{array}$ & $\begin{array}{l}\text { direction } \\
\varphi \operatorname{cd}\left({ }^{\circ}\right)\end{array}$ & $\begin{array}{l}\text { Count-rate } \\
\left(\times 10^{3} / \mathrm{hr}\right) \\
\end{array}$ & $\begin{array}{l}\text { Median primary } \\
\text { energy }(\mathrm{GeV})\end{array}$ \\
\hline E-telescope & $\mathrm{NE}$ & 35 & 38 & 4.2 & 773 \\
\hline$(E=N E+S E)$ & S E & 35 & 128 & 4.5 & 776 \\
\hline W-telescope & NW & 35 & 308 & 7.1 & 620 \\
\hline$(\mathrm{W}=\mathrm{NW}+\mathrm{SW})$ & S W & 35 & 218 & 6.7 & 649 \\
\hline
\end{tabular}

$\lambda c d$ and $\varphi c d$ represent the asymptotic latitude and longitude in geographic coordinate system of center direction of the telescope.

Table 2. Earth's motion Compton-Getting effect for Q- and A-period; the observed E-W and its rotated vectors in a clockwise by $90^{\circ}$. The expected C-G vector is estimated by the coupling coefficients for a flat variational spectrum in a whole energy range (FujImoto et al., 1984).

\begin{tabular}{cccc}
\hline & \multicolumn{2}{c}{$\begin{array}{c}\text { Observed } \\
\text { (after rotation) }\end{array}$} & Expected \\
\hline Q-period & A. $=0.030 \pm 0.007 \%$ & A. $=0.030 \pm 0.007 \%$ & A. $=0.021 \%$ \\
& Ph. $=22.5 \pm 1.3 \mathrm{hr} \mathrm{LT}$ & $\mathrm{Ph} .=4.5 \pm 1.3 \mathrm{hr} \mathrm{LT}$ & Ph. $=6.0 \mathrm{hr} \mathrm{LT}$ \\
A-period & A. $=0.009 \pm 0.006 \%$ & A. $=0.009 \pm 0.006 \%$ & - \\
& $\mathrm{Ph} .=6.0 \pm 3.5 \mathrm{hr} \mathrm{LT}$ & $\mathrm{Ph} .=12.0 \pm 3.5 \mathrm{hr} \mathrm{LT}$ & - \\
\hline
\end{tabular}




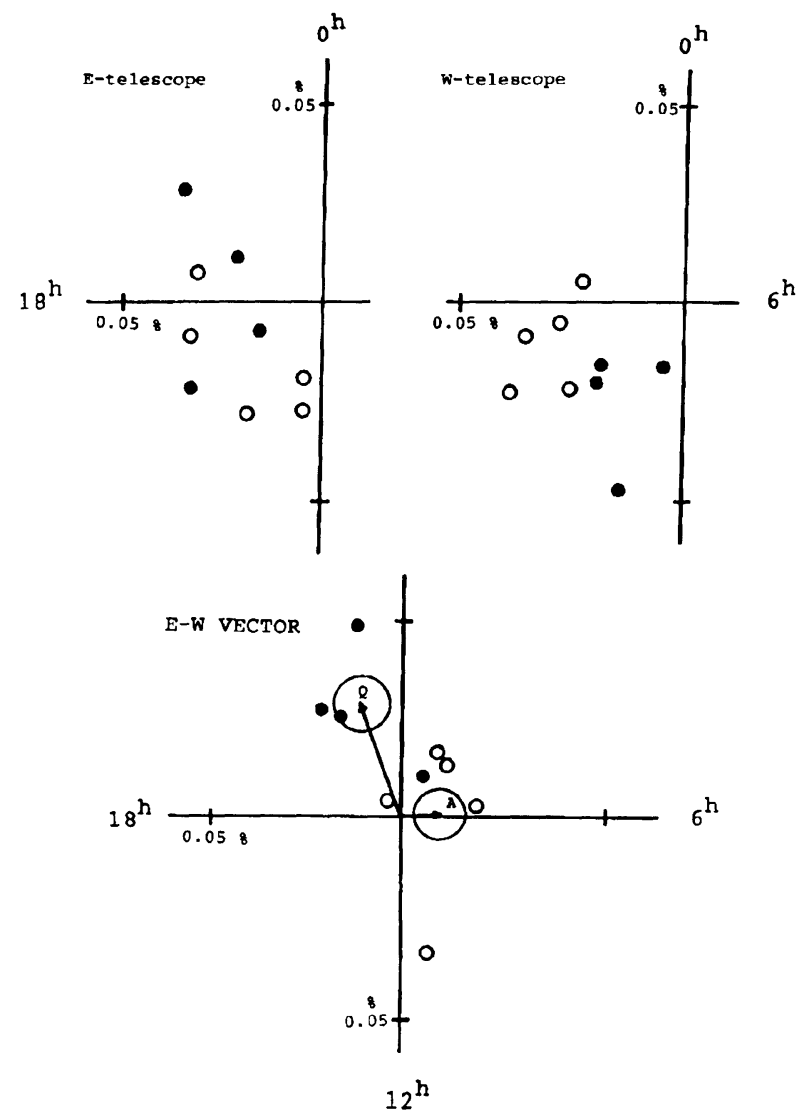

Fig. 1. Yearly solar diurnal vectors for E- and W-telescope and their difference E-W vectors in the period 1980-1989; filled circles for Q-period and open circles for A-period. Averaged E-W vectors are also shown. The error circles are derived from dispersion of yearly vectors.

Among those vectors in Eq. (2), we can eliminate $\boldsymbol{D}$ (TEMP) by taking the difference between $\mathrm{E}$ - and W-telescope as was done in the previous section. The E-W vectors thus obtained (containing $D(C R)$ and $D(C G)$ ) are free from the atmospheric effects and may reflect the cosmic ray modulation in space. These are again plotted (with solid line) in Fig. 3 for both Q- and A-period. It is found in Table 2 that for Q-period the averaged E-W vector has the phase of $22.5 \pm 1.3 \mathrm{hr}$ LT and the amplitude of $0.030 \pm 0.07 \%$ and for A-period the averaged E-W vector has the phase of $6.0 \pm 3.5 \mathrm{hr}$ LT with the amplitude of $0.009 \pm 0.006 \%$, respectively. To compare these observed vectors with the expected $\mathrm{C}-\mathrm{G}$ effect, we rotate the E-W vectors (solid line) by the right angle in a clockwise, based on its geometrical arrangement of E- and W-telescope (see Table 1). In Fig. 3, the rotated vectors are plotted with the dotted line and the expected $\mathrm{C}-\mathrm{G}$ effect is shown with the term of $\mathrm{C}-\mathrm{G}$ effect in the figure. The expected C-G vector may be estimated, based on Eq. (1) and by taking into accounts the coupling coefficients (FUJIMOTO et al., 1984) for a flat variational spectrum in a 


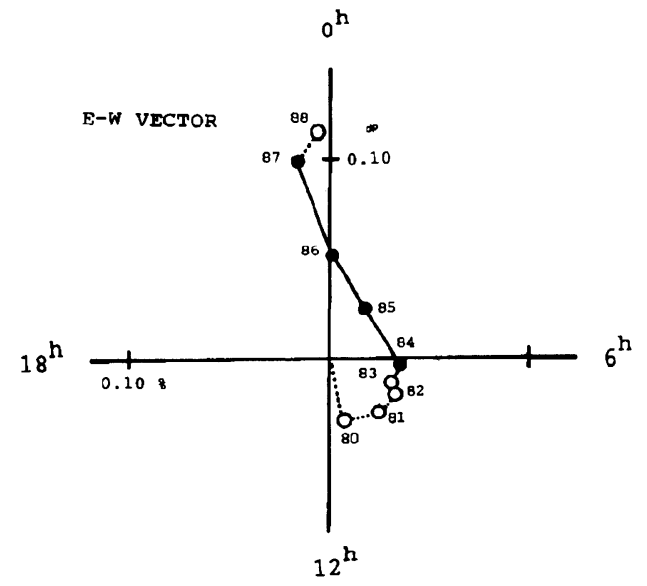

Fig. 2. Sum harmonic dial of the E-W vectors shown in Fig. 1, connected with the solid line during Qperiod (filled circles) and with the dotted line during A-period (open circles) for the period 19801989. The number of each point represents the year (e.g., 80 represents the year 1980).

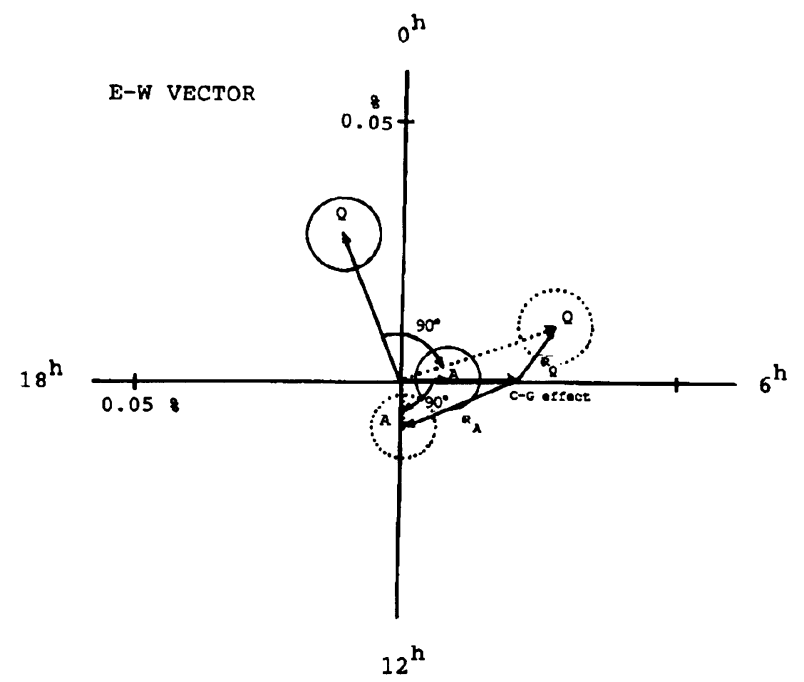

Fig. 3. Observed E-W vectors (solid line) and their rotated vectors in a clockwise by $90^{\circ}$ (dotted line) for both Q- and A-period. The expected C-G vector is also shown. $\boldsymbol{R}_{\mathrm{Q}}$ for Q-period and $\boldsymbol{R}_{\mathrm{A}}$ for A-period are the difference vectors between the observed and the $\mathrm{C}-\mathrm{G}$ vector, respectively.

whole energy range. We can see in Fig. 3 and Table 2 that for Q-period the observed and the expected vectors ( $\sim 0.02 \%$ amplitude in $\sim 6 \mathrm{hr}$ LT phase) are in fair agreement with each other with a small difference between them. At the present moment, this discrepancy is not assignable to the known sources, but the difference vector $\boldsymbol{R}_{\mathrm{Q}}$ for Q-period may be regarded as being mainly due to a small sample of the present data for 4-year period. On the other hand, for A-period, the rotated E-W vector is found to be shifted to later hours in around $12 \mathrm{hr}$ LT. We can see in the fiqure that the difference vector $\boldsymbol{R}_{\mathrm{A}}$ for A-period may be directed to around 
$16 \mathrm{hr} \mathrm{LT}$ and would correspond to the diurnal vector $\boldsymbol{D}(\mathrm{CR})$ due to solar anisotropy, which is consistent with those in the lower energy regions (e.g. KUDO et al., 1987). It may be concluded that for the last solar active period, the upper cut-off energy of modulation of solar diurnal anisotropy is as high as that (several $10^{11} \mathrm{eV}$ ) for the threshold $\left(\sim 7 \cdot 10^{10} \mathrm{eV}\right)$ of 220 m.w.e. depth underground. It is added to note that $D$ (TEMP) may also be determined for Qperiod, based on Eq. (2) and the fact that $D(C R)$ may be diminished during this period due to the lower cut-off energy of modulation $\left(\sim 5 \cdot 10^{10} \mathrm{eV}\right)$ (e.g. KUDO et al., 1987) and also the C$\mathrm{G}$ effect is time invariant because of the effect due to the Earth's movement. The D(TEMP) obtained in the present analysis $(\sim 17 \mathrm{hr}$ LT phase and $\sim 0.05 \%$ amplitude) is in good agreement with our previous result ( $\sim 17 \mathrm{hr}$ LT phase and $\sim 0.033 \%$ amplitude) for another Matsushiro underground station (220 m.w.e. depth) (MORI et al., 1988).

In conclusion, we can summarize the present results at Matsushiro underground station as follows:

1) For the last quiet period, we observe the Earth's motion Compton-Getting effect using the underground muon data with a difference between E- and W-telescope.

2) For the last active period, the observed solar diurnal vector may indicate some contribution from solar diurnal anisotropy, in addition to the $\mathrm{C}-\mathrm{G}$ effect on it. This suggests that the upper cut-off energy of modulation may be as high as that (several $10^{11} \mathrm{eV}$ ) for the threshold of 220 m.w.e. depth underground.

The authors express their great appreciation to Profs. S. Sagisaka and M. Ichinose for their kind discussions. Thanks are also due to Messrs. K. Chino, S. Akahane, T. Itoh and to our colleagues of Shinshu University for their kind help in routine works and data processings.

\section{REFERENCES}

Compton, A. H. and I. A. Getring, An apparent effect of galactic rotation on the intensity of cosmic rays, Phys. Rev., 47, 817-821, 1935.

Cutler, D. J. and D. E. Groom, Observation of terrestrial orbital motion using the cosmic-ray ComptonGetting effect, Nature, 323, 434-436, 1986.

Davies, S. T., H. Elliot, R. G. Marsden, T. Thambyahpillai, and J. C. Dutt, The angular distribution of cosmic rays at the boundary of the heliosphere, Planet. Space Sci., 27, 733-738, 1979.

Fuji, Z. and H. Ueno, Long-term change in the cosmic ray diurnal variation, Proc. 21st Int. Conf. Cosmic Rays (Adelaide), 6, 314-317, 1990.

Fujimoto, K., A. Inoue, K. Murakami, and K. Nagashima, Coupling coefficients of cosmic ray daily variations for meson telescopes, Rep. Cosmic-Ray Res. Lab., Nagoya Univ., No. 9, 1984.

Kudo, S., S. Mori, D. B. Swinson, S. Yasue, and J. Y. RyU, Solar cycle dependence of solar diurnal anisotropy, Proc. 20th Int. Conf. Cosmic Rays (Moscow), 4, 140-143, 1987.

MoRI, S., S. SAGISAKA, and S. YASUE, The atmospheric temperature effect on the diurnal variation of cosmicray muon intensity observed at 220 m.w.e. underground, J. Geomag. Geoelectr., 40, 1023-1033, 1988.

Nagashima, K., K. Fujimoto, S. Sakakibara, Z. Fujit, H. Ueno, I. Morishita, and K. Murakami, Galactic cosmic-ray anisotropy and its modulation in the heliomagnetosphere, inferred from air shower observation at Mt. Norikura, Nuovo Cimento, 12C, 695-749, 1989.

Yasue, S., S. Mori, M. Ichinose, S. Sagisaka, T. Yokoyama, S. AKahane, and K. Chino, the observation of cosmic ray intensity variation at 250 m.w.e. underground at matsushiro, Proc. 16th Int. Conf. Cosmic Rays (Kyoto), 4, 227-231, 1979.

Yasue, S., S. Mori, S. SAgisaka, and M. Ichinose, Sidereal diurnal variation observed at Matsushiro, Proc. Int. Symp. Cosmic Rays (Morioka), 355-359, 1984.

Yasue, S., S. Mori, and S. SAgisaKa, Cosmic ray intensity variations observed at Matsushiro (220 m.w.e. in depth), Proc. 19th Int. Conf. Cosmic Rays (La Jalla), 5, 56-59, 1985. 